\title{
Distortion risk measures for sums of dependent losses
}

\author{
Brahim Brahimi, Djamel Meraghni, and Abdelhakim Necir \\ Laboratory of Applied Mathematics, Mohamed Khider University of Biskra, 07000, Algeria
}

Received 29 June 2010; Accepted 25 October 2010

Copyright (C) 2010, Journal Afrika Statistika. All rights reserved

\begin{abstract}
We discuss two distinct approaches, for distorting risk measures of sums of dependent random variables, which preserve the property of coherence. The first, based on distorted expectations, operates on the survival function of the sum. The second, simultaneously applies the distortion on the survival function of the sum and the dependence structure of risks, represented by copulas. Our goal is to propose risk measures that take into account the fluctuations of losses and possible correlations between risk components.

Résumé. Nous discutons deux approches distinctes, de distortion des mesures de risque de la somme de variables aléatoires dépendantes, qui conservent la propriété de cohérence. La première, basée sur les espérances distordues, agit sur la fonction de survie de la somme. La seconde, applique des déformations simultanées sur la fonction de survie de la somme et sur la structure de dépendance des risques, représentée par une copule. Notre objectif est de proposer des mesures qui prennent en compte les fluctuations des pertes et des corrélations éventuelles entre les composantes d'un risque multivarié.
\end{abstract}

Key words: Coherence; Dependence structure; Distortion function; Risk measure; Risk theory; insurance; Wang transform.

AMS 2010 Mathematics Subject Classification : 60B05, 62H20, 91B30.

\section{Introduction}

Risk measures are used to quantify insurance losses and financial assessments. Several risk measures have been proposed in actuarial science literature, namely: the Value-at-Risk (VaR), the expected shortfall or the conditional tail expectation (CTE), and the distorted risk measures (DRM). Before introducing and interpreting the DRM, it is necessary to fix a convention of profit and loss appropriate to the application to the market finance, the credit risk and to the insurance. Let $X$ be a random variable (rv), representing losses (or gains) of a company, with a continuous distribution function (df) $F$. The DRM of rv $X$, due to Wang [17], is defined as follows:

$$
\pi_{\psi}[X]:=\int_{0}^{\infty} \psi(1-F(x)) d x,
$$

where $\psi$ is a non-decreasing function, called distortion function, satisfying $\psi(0)=0$ and $\psi(1)=1$. In the actuarial literature the following functions are frequently used:

$$
\begin{array}{ll}
\psi_{\rho}(s)=s^{\rho}, & \text { for } 0<\rho \leq 1, \\
\psi_{\kappa}(s)=\phi\left(\phi^{-1}(s)+\kappa\right), & \text { for } 0 \leq \kappa<\infty, \\
\psi_{\zeta}(s)=\min (s /(1-\zeta), 1) & \text { for } 0 \leq \zeta<1, \\
\psi_{\alpha}(s)=s^{\alpha}(1-\alpha \ln s), & \text { for } 0<\alpha \leq 1,
\end{array}
$$

Brahim Brahimi: brah.brahim@gmail.com

Djamel Meraghni: dmeraghni@yahoo.fr

Abdelhakim Necir: ah.necir@univ-biskra.dz 
where $\phi^{-1}(u):=\inf \{x: \phi(x) \geq u\}$ is the quantile function of the standard normal distribution $\phi$. Constants $\rho, \kappa, \zeta$ and $\alpha$ are called distortion parameters. The functions $\psi_{\rho}, \psi_{\kappa}, \psi_{\zeta}$ and $\psi_{\alpha}$ respectively give rise to the so-called proportional hazard transform (PHT) (Wang [17]), the normal transform (Wang [18]), the CTE and the look-back distortion (Hürlimann [12]). When $\rho=1$ and $\kappa=\zeta=0$, there is no distortion and the corresponding DRM is equal to the expectation of $X$. For recent literature on risk measures one refers to Denuit et al. [6] and Furman and Zitikis ([9], $[10])$.

The problem of the axiomatic foundation of risk measures has received much attention starting with the seminal paper of Artzner et al. [1], where the definition of coherent risk measure was first provided. A coherent risk measure is a real functional $\mu$, defined on a space of rv's, satisfying the following axioms:

H1. Boundedness from above by the maximum loss: $\mu(X) \leq \max (X)$.

H2. Boundedness from below by the mean loss: $\mu(X) \geq \mathbb{E}(X)$.

H3. Scalar additivity and multiplicativity : $\mu(a X+b)=a \mu(X)+b$, for $a, b \geq 0$.

H4. Subadditivity: $\mu(X+Y) \leq \mu(X)+\mu(Y)$.

The only axiom that a DRM may lack in order to be a coherent risk measure in the sense of Artzner et al. [1] is H4. However, the subadditivity theorem of Choquet integrals (Denneberg [5]) guarantees that $\mu(X+Y) \leq \mu(X)+\mu(Y)$ if and only if the distortion function $\psi$ is concave. Hence, the DRM $\pi_{\psi}[X]$ defined in (1) with a concave distortion $\psi$ is coherent. It is well known that the CTE and the PHT are examples of concave distortion risk measures, whereas the VaR is not. In traditional risk theory, individual risks have been usually assumed to be independent. Traceability

for this assumption is very convenient, but not realistic. Recently in the actuarial science, the study of the impact of dependence among risks has become a major and flourishing topic. Several notions of dependence were introduced to model the fact that larger values of one component of a multivariate risk tend to be associated with larger values of the others. In this paper, we deal with a vector of risk losses $\mathbf{X}=\left(X^{(1)}, \ldots, X^{(d)}\right), d \geq 2$ and we discuss the computation of the DRM of the sum $Z$ of its components. When $X^{(1)}, \ldots, X^{(d)}$ are independent and identically distributed, their sum is considered as a rv whose df $G$ is the convolution of the marginal distributions of $\mathbf{X}$. In this case, the DRM value of $Z$, for a given distortion function $\psi$ may be obtained via formula (1), that is

$$
\pi_{\psi}[Z]:=\int_{0}^{\infty} \psi(1-G(z)) d z .
$$

Now, assume that $X^{(1)}, \ldots, X^{(d)}$ are dependent with joint $\mathrm{df} H$ and continuous margins $F_{i}, i=1, \ldots, d$. In this case, the problem becomes different and its resolution requires more than the usual background. Several authors discussed the DRM, when applied to sums of rv's, against some classical dependency measures such as Person's $r$, Spearman's $\rho$ and Kendall's $\tau$, see for instance, Darkiewicz et al. [4] and Burgert and Rüschendorf [2]. Our contribution is to introduce the copula notion to provide more flexibility to the DRM of sums of rv's in terms of loss and dependence structure. For comprehensive details on copulas one may consult the textbook of Nelsen [14]. According to Sklar's Theorem (Sklar $[15])$, there exists a unique copula $C:[0,1]^{d} \rightarrow[0,1]$ such that

$$
H\left(x_{1}, \ldots, x_{d}\right)=C\left(F_{1}\left(x_{1}\right), \ldots, F_{d}\left(x_{d}\right)\right)
$$

The Copula $C$ is the joint df of rv's $U_{i}=F_{i}\left(X^{(i)}\right), i=1, \ldots, d$. It is defined on $[0,1]^{d}$ by $C\left(u_{1}, \ldots, u_{d}\right)=$ $H\left(F_{1}^{-1}\left(u_{1}\right), \ldots, F_{d}^{-1}\left(u_{d}\right)\right)$, where $F_{i}^{-1}$ denotes the quantile function of $F_{i}$. This means that the DRM of the sum is a functional of both copula $C$ and margins $F_{i}$. Therefore, one must take into account the dependence structure and the behavior of margin tails. These two aspects have an important influence when quantifying risks. If the correlation factor is neglected, the calculation of the DRM follows formula (2), which only focuses on distorting the tail. In order to highlight the dependence structure, we add a distortion on the copula as well. The notion of distorted copula has recently been considered by several authors, see for instance Frees and Valdez [8], Genest and Rivest [11], Morillas [13], Crane and van der Hoek [3] and Valdez and Xiao [16]. Given a copula $C$ and a non-decreasing bijection $\Gamma:[0,1] \rightarrow[0,1]$, the distorted copula $C^{\Gamma}$ is defined by

$$
C^{\Gamma}\left(u_{1}, \ldots, u_{d}\right):=\Gamma^{-1}\left(C\left(\Gamma\left(u_{1}\right), \ldots, \Gamma\left(u_{d}\right)\right)\right) .
$$

This transformation will affect the joint df $H$ and consequently the $\operatorname{df} G$ of the sum $Z$. Their new forms will be denoted by $H^{\Gamma}$ and $G^{\Gamma}$ respectively. Morillas [13] describes some of the existing families of distortion functions, among which 
the following are frequently used:

$$
\begin{aligned}
& \Gamma_{r}(s)=s^{r}, \quad \text { for } \quad 0<r \leq 1, \\
& \Gamma_{\delta}(s)=\frac{\ln (\delta s+1)}{\ln (\delta+1)}, \text { for } \quad \delta>0, \\
& \Gamma_{\xi, \vartheta}(s)=\frac{(\xi+\vartheta) s}{\xi s+\vartheta}, \text { for } \quad \xi, \vartheta>0 \\
& \Gamma_{\nu}(s)=\frac{s^{\nu}}{2-s^{\nu}}, \quad \text { for } 0<\nu \leq 1 / 3 .
\end{aligned}
$$

We call the corresponding distorted risk measures by copula distorted risk measure (CDRM) defined as

$$
\pi_{\psi}^{\Gamma}[Z]=\int_{0}^{\infty} \psi\left(1-G^{\Gamma}(z)\right) d z
$$

It is worth mentioning that if $X^{(1)}, \ldots, X^{(d)}$ are independent, the corresponding copula function $C\left(u_{1}, \ldots, u_{d}\right)=\prod_{i=1}^{d} u_{i}$ is called the product copula and denoted by $C^{\perp}$. In this case, we have $C^{\Gamma}=C$ and therefore $\pi_{\psi}^{\Gamma}[Z]=\pi_{\psi}[Z]$.

The remainder of this paper is organized as follows. In Section 2, we give a copula representation of the DRM's. In Section 3, we present a more flexible class of copula given by the notion of distorted Archimedean copulas. By the nice properties of this class and the copula representation of the DRM, we introduce, in Section 4, the CDRM's. Finally, an illustrative example, explaining the CDRM computation, is given in Section 5.

\section{Copula representation of the DRM}

Given a vector of risk losses $\mathbf{X}=\left(X^{(1)}, \ldots, X^{(d)}\right), d \geq 2$, with joint $\mathrm{df} H$ and continuous margins $F_{i}, i=1, \ldots, d$. The df of the $\mathrm{rv} Z=\sum_{i=1}^{d} X_{i}$, is

$$
G(t)=\int_{A(t)} d H\left(x_{1}, . ., x_{d}\right), \text { for any } t \geq 0,
$$

where $A(t):=\left\{\left(x_{1}, . ., x_{d}\right): 0 \leq \sum_{i=1}^{d} x_{i} \leq t\right\}$. Using the representation (3), we get

$$
G(t)=\int_{A(t)} d C\left(F_{1}\left(x_{2}\right), \ldots, F_{d}\left(x_{d}\right)\right) .
$$

If we suppose that the copula $C$ and margins $F_{i}$ are differentiable with densities $c$ and $f_{i}$, respectively, then

$$
G(t)=\int_{A(t)} c\left(F_{1}\left(x_{1}\right), \ldots, F_{d}\left(x_{d}\right)\right) \prod_{i=1}^{d} f_{i}\left(x_{i}\right) d x_{1}, \ldots d x_{d}
$$

The change of variables $F_{i}\left(x_{i}\right)=u_{i}, i=1, \ldots, d$, yields

$$
G(t)=\int_{0}^{F_{d}(t)} \int_{0}^{F_{d-1}\left(t-F_{d}^{-1}\left(u_{d}\right)\right)} \ldots \int_{0}^{F_{1}\left(t-\sum_{i=0}^{d-2} F_{d-i}^{-1}\left(u_{d-i}\right)\right)} c\left(u_{1}, \ldots, u_{d}\right) d u_{1} \ldots d u_{d} .
$$

According to (4), the computation of the DRM corresponding to $Z$, given in (2), requires the knowledge of the copula density and the margins of vector $\mathbf{X}$. In particular, for the bivariate case $(d=2)$, we have

$$
G(t)=\int_{0}^{F_{2}(t)} \int_{0}^{F_{1}\left(t-F_{2}^{-1}\left(u_{2}\right)\right)} c\left(u_{1}, u_{2}\right) d u_{1} d u_{2} .
$$

Whenever $X_{1}$ and $X_{2}$ are independent, we have $c\left(u_{1}, u_{2}\right)=1$, and therefore

$$
G(t)=\int_{0}^{F_{2}(t)} F_{1}\left(t-F_{2}^{-1}\left(u_{2}\right)\right) d u_{2}=\int_{0}^{t} F_{1}(t-x) d F_{2}(x),
$$

which is the usual convolution of the $F_{i}$ 's. 


\section{Distorted Archimedean copulas}

In this paper, we focus on one important class of copulas called: Archimedian copulas. This class contains several copula families useful in dependence modelling. Their nice properties are captured by an additive generator function $\varphi:[0,1] \rightarrow[0, \infty]$, which is continuous, strictly decreasing and convex with $\varphi(1)=0$. The main advantage of the Archimedean copulas is the achievement of the reduction in dimensionality of a $d$-variate distribution in a single argument. In econometrics, this property has the potential to be of use in models of limited dependent variables, especially those requiring some probabilistic enumeration on high-dimensional subspaces. In the bivariate case, an Archimedean copula is defined by

$$
C(u, v)=\varphi^{[-1]}(\varphi(u)+\varphi(v))
$$

where

$$
\varphi^{[-1]}(t)= \begin{cases}\varphi^{-1}(t), & 0 \leq t \leq \varphi(0), \\ 0, & \varphi(0) \leq t \leq \infty .\end{cases}
$$

Note that $\varphi^{[-1]}$ is continuous and non-increasing on $[0, \infty]$ and $\varphi$ is the unique generator up to a scaling constant. If the terminal $\varphi(0)=\infty$, the generator is called strict and $\varphi^{[-1]}=\varphi^{-1}$. Numerous single-parameter families of Archimedean copulas are listed in Table 4.1 in Nelsen [14]. Particular examples are $\varphi_{\theta}(t)=\left(t^{-\theta}-1\right) / \theta, \varphi_{\alpha}(t)=(-\ln t)^{\alpha}$ and $\varphi_{\beta}(t)=-\ln \left(\left(e^{-\beta t}-1\right) /\left(e^{-\beta}-1\right)\right)$ which are, respectively, the generators of the Clayton family

$$
C_{\theta}(u, v)=\left(u^{-\theta}+v^{-\theta}-1\right)^{-1 / \theta}, \theta \geq 0,
$$

the Gumbel family

$$
C_{\alpha}(u, v)=\exp \left\{-\left[(-\ln u)^{\alpha}+(-\ln v)^{\alpha}\right]^{1 / \alpha}\right\}, \alpha \geq 1
$$

and the Frank family

$$
C_{\beta}(u, v)=-\frac{1}{\beta} \ln \left[1+\frac{\left(e^{\beta u}-1\right)\left(e^{\beta v}-1\right)}{e^{\beta}-1}\right], \beta \in \mathbb{R} \backslash\{0\} .
$$

The generators $\varphi_{\theta}, \varphi_{\alpha}$ and $\varphi_{\beta}$ are strict and therefore their corresponding copulas $C_{\theta}, C_{\alpha}$ and $C_{\beta}$ verify

$$
C(u, v)=\varphi^{-1}(\varphi(u)+\varphi(v))
$$

Next, we discuss some properties of distortion functions acting on bivariate Archimedean copulas. Given an Archimedean copula $C$ and a strictly increasing bijection $\Gamma:[0,1] \rightarrow[0,1]$, we consider the function $C^{\Gamma}:[0,1]^{2} \rightarrow[0,1]$ defined by

$$
C^{\Gamma}(u, v)=\Gamma^{-1}(C(\Gamma(u), \Gamma(v))) \text {. }
$$

Under what conditions on $\Gamma$, the function $C^{\Gamma}$ is an Archimedean copula?

First, from Theorem 3.3.3. in Nelsen [14], $C^{\Gamma}$ is a copula if $\Gamma$ is concave and continuous on $[0,1]$ with $\Gamma(0)=0$ and $\Gamma(1)=1$. The following Theorem gives an additional condition so that the copula $C^{\Gamma}$ remains Archimedean. For convenience, let $\mathbb{K}$ represents the set of the functions $\Gamma$ verifying the assumptions above.

Theorem 1. Let $C$ be an Archimedean copula with generator $\varphi$ and suppose that $\Gamma \in \mathbb{K}$, then the copula $C^{\Gamma}$ is Archimedean if and only if $\varphi \circ \Gamma$ is convex.

Proof. Indeed, let $\varphi$ be the generator of the copula $C$ and let $\Gamma \in \mathbb{K}$, then

$$
C^{\Gamma}\left(u_{1}, \ldots, u_{d}\right)=\Gamma^{-1}\left(C\left(\Gamma\left(u_{1}\right), \ldots, \Gamma\left(u_{d}\right)\right)\right) .
$$

We have $\Gamma^{[-1]}=\Gamma^{-1}$, then

$$
C^{\Gamma}\left(u_{1}, \ldots, u_{d}\right)=\Gamma^{[-1]} \varphi^{[-1]}\left(\varphi\left(\Gamma\left(u_{1}\right)\right)+\ldots+\varphi\left(\Gamma\left(u_{d}\right)\right)\right) .
$$

It is easy to show that $\Gamma^{[-1]} \varphi^{[-1]}=(\varphi \circ \Gamma)^{[-1]}$, it follows that

$$
C^{\Gamma}\left(u_{1}, \ldots, u_{d}\right)=\mathcal{T}^{[-1]}\left(\mathcal{T}\left(u_{1}\right)+\ldots+\mathcal{T}\left(u_{d}\right)\right)
$$

with $\mathcal{T}:=\varphi \circ \Gamma$. From Theorem 4.1.4. Nelsen [14], $C^{\Gamma}$ is Archimedean if and only if $\mathcal{T}$ is convex. Notice that $\varphi \circ \Gamma$ is the generator of $C^{\Gamma}$.

Corollary 1. The distortion function $t \rightarrow \Gamma^{\perp}(t):=\exp (-\varphi(t))$ transforms any Archimedean copula $C$ in the product copula $C^{\perp}$. 
Proof. Straightforward.

Next, we see the influence of the distortion of copulas on the association measures. Kendall's tau and Spearman's rho are the most popular measures of association, their representations in terms of the copula $C$ are given by

$$
\tau=4 \int_{0}^{1} \int_{0}^{1} C(u, v) d C(u, v)-1 \text { and } \rho=12 \int_{0}^{1} \int_{0}^{1}(C(u, v)-u v) d u d v
$$

respectively. Let $\tau^{\Gamma}$ and $\rho^{\Gamma}$, respectively, denote Kendall's tau and Spearman's rho of copula $C^{\Gamma}$. According to Theorem 10 in Durrleman et al. [7], we have under suitable assumptions

$$
1+\frac{\tau-1}{a^{2}} \leq \tau^{\Gamma} \leq 1+\frac{\tau-1}{b^{2}}
$$

and

$$
\frac{\rho+3}{a^{3}}-3 \leq \rho^{\Gamma} \leq \frac{\rho+3}{b^{3}}-3
$$

where $0<a \leq b<\infty$ are bounds for the derivative of $\Gamma$.

\section{Risk measures for sums of losses}

It may happen that the model (represented by the copula $C$ ) chosen, to fit the data, does not provide enough information on the dependence structure. This leads us to transform $C$ to a more flexible copula $C^{\Gamma}$ of the same class. Consequently, the joint df of $\mathbf{X}$ may be represented, via Sklar's Theorem, as

$$
H\left(x_{1}, \ldots, x_{d}\right)=C^{\Gamma}\left(F_{1}\left(x_{1}\right), \ldots, F_{d}\left(x_{d}\right)\right) .
$$

Suppose that $C$ is Archimedean with generator $\varphi$, then from Theorem 1, $C^{\Gamma}$ defined in (5) is also Archimedean. Assume that $C^{\Gamma}$ has a density function $c^{\Gamma}$, then in view of the representation (4) the $\operatorname{df} G^{\Gamma}$ of the sum $Z$ may be written as

$$
G^{\Gamma}(t):=\int_{0}^{F_{d}(t)} \int_{0}^{F_{d-1}\left(t-F_{d}^{-1}\left(u_{d}\right)\right)} \ldots \int_{0}^{F_{1}\left(t-\sum_{i=0}^{d-2} F_{d-i}^{-1}\left(u_{d-i}\right)\right)} c_{\Gamma}\left(u_{1}, \ldots, u_{d}\right) d u_{1} \ldots d u_{d} .
$$

Applying Wang's principle (1) to the loss distribution $G^{\Gamma}$, we have

$$
\pi_{\psi}^{\Gamma}[Z]:=\int_{0}^{+\infty} \psi\left(1-G^{\Gamma}(t)\right) d t
$$

which we call the CDRM. This may be considered as a manner of measuring the risk $Z$ by distorting both the dependence structure and the distribution tail, without losing the coherence feature. The CDRM adjusts the true probability measure to give more weight to higher risk events and less weight to the dependence structure. In other words, the simultaneous transformations yield a new risk measure bounded by the expectation and Wang's measure, that is

$$
\mathbb{E}[Z] \leq \pi_{\psi}^{\Gamma}[Z] \leq \pi_{\psi}[Z]
$$

In the following example, we verify the previous inequalities on a selected model.

\section{Illustrative example}

Let $X_{1}$ and $X_{2}$ be two risks with joint df represented by the Clayton copula $C_{\theta}, \theta>0$ and Pareto-distributed margins $F_{1}$ and $F_{2}$ with respective parameters $0<\alpha_{1}, \alpha_{2}<1$, that is $F_{i}\left(x_{i}\right)=1-x_{i}^{-1 / \alpha_{i}}, x_{i}>1, i=1,2$. Kendall's tau of $C_{\theta}$ is $\tau=\theta /(\theta+2)$. Let $\psi(x)=x^{1 / \rho}, \rho \geq 1$, and $\Gamma(t)=t^{1 / \delta}, \delta \geq 1$. The distorted copula $C_{\theta}^{\Gamma}$, denoted by $C_{\theta}^{\delta}$, is of Clayton type with generator $(\varphi \circ \Gamma)(t)=\left(t^{-\theta / \delta}-1\right) / \theta$ and Kendall's tau is $\tau^{\Gamma}=(\theta / \delta) /(\theta / \delta+2)$. The df of the sum $Z=X_{1}+X_{2}$ is

$$
G^{\delta}\left(t ; \theta, \alpha_{1}, \alpha_{2}\right)=\int_{1}^{1-t^{-1 / \alpha_{2}}}\left(\int_{1}^{1-\left(t-(1-v)^{-\alpha_{2}}\right)^{-1 / \alpha_{1}}} c_{\theta}^{\delta}(u, v) d u\right) d v
$$

where

$$
c_{\theta}^{\delta}(u, v)=(\theta / \delta+1) u^{-\theta / \delta-1} v^{-\theta / \delta-1}\left(u^{-\theta / \delta}+v^{-\theta / \delta}-1\right)^{-\delta / \theta-2},
$$

is the density of $C_{\theta}^{\delta}$. Figure 1 gives a preview of the effect of the copula distortion. 

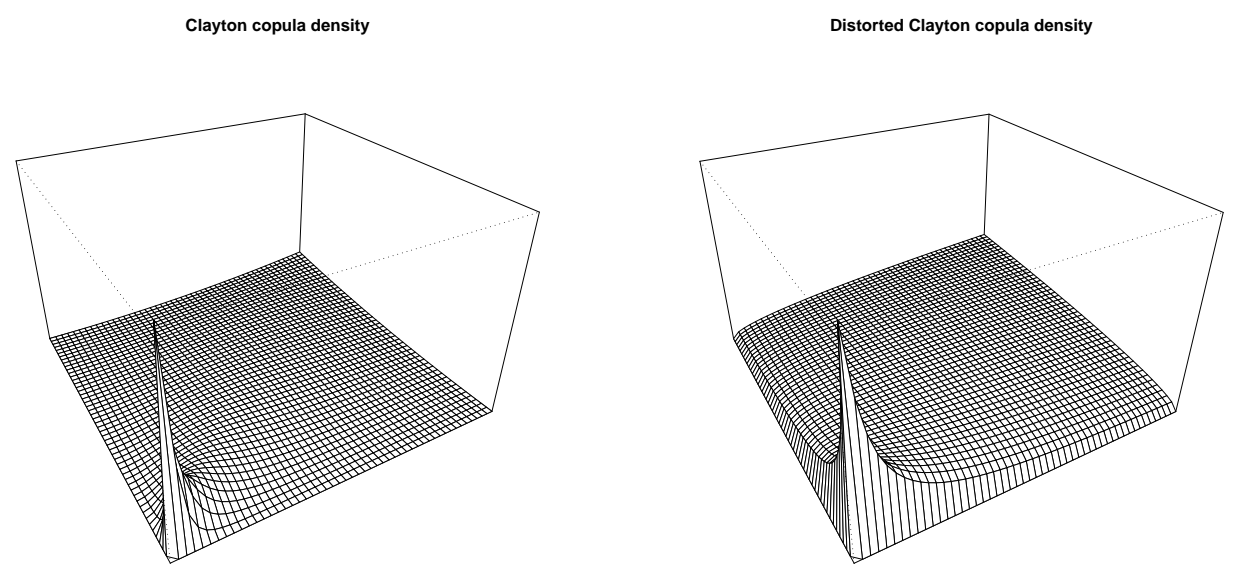

Figure 1. Clayton copula density with $\theta=2$ (left panel) and its distorted copula density with $\delta=4$ (right panel)

The DRM and the CDRM of $Z$ are respectively denoted by

$$
\pi_{\rho}[Z]=\int_{2}^{\infty}(1-G(t))^{1 / \rho} d t, \quad \text { and } \quad \pi_{\rho}^{\delta}[Z]=\int_{2}^{+\infty}\left(1-G^{\delta}(t)\right)^{1 / \rho} d t
$$

We select a Pareto model with $\theta=3 / 2, \alpha_{1}=1 / 3$ and $\alpha_{2}=1 / 5$. We obtain $\mathbb{E}(Z)=0.750$ and $\tau=0.428$. For two different tail distortion parameters $\rho=1.2$ and $\rho=1.4$ the respective DRM's are 1.225 and 2.091. The CDRM's for distinct values of the copula distortion parameter $\delta$ are summarized in Tables 1 and 2, where we see that the inequalities (6) are satisfied for any value of the copula distortion parameter. This is well shown graphically in Figure 2 in which the three risk measures of (6) are plotted as functions of $\delta$.

\begin{tabular}{l|ccccccccc}
\hline$\delta$ & 1 & 1.5 & 2 & 2.5 & 3 & 3.5 & 4 & 5 & 6 \\
\hline \hline$\tau^{\delta}$ & 0.428 & 0.333 & 0.272 & 0.230 & 0.200 & 0.176 & 0.157 & 0.130 & 0.111 \\
$\pi_{\rho}^{\delta}[Z]$ & 1.225 & 1.030 & 0.988 & 0.969 & 0.964 & 0.961 & 0.958 & 0.953 & 0.950 \\
\hline \hline
\end{tabular}

Table 1. CDRM's and transformed Kendall tau of the sum of two Pareto-distributed risks with tail distortion parameter $\rho=1.2$.

\begin{tabular}{l|ccccccccc}
\hline$\delta$ & 1 & 1.5 & 2 & 2.5 & 3 & 3.5 & 4 & 5 & 6 \\
\hline \hline$\tau^{\delta}$ & 0.428 & 0.333 & 0.272 & 0.230 & 0.200 & 0.176 & 0.157 & 0.130 & 0.111 \\
$\pi_{\rho}^{\delta}[Z]$ & 2.091 & 1.801 & 1.736 & 1.712 & 1.703 & 1.699 & 1.694 & 1.685 & 1.680 \\
\hline \hline
\end{tabular}

Table 2. CDRM's and transformed Kendall tau of the sum of two Pareto-distributed risks with tail distortion parameter $\rho=1.4$. 


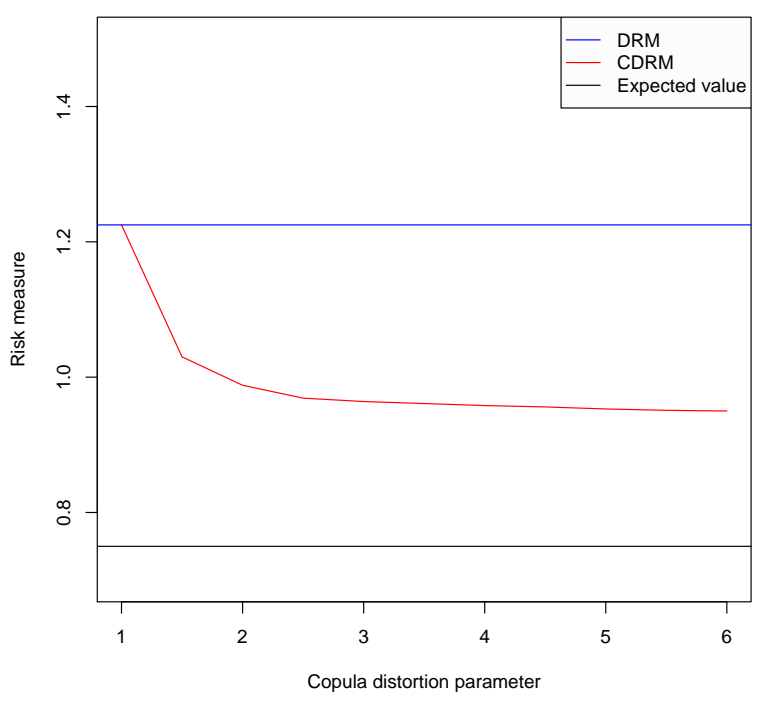

Figure 2. Risk measures of the sum of two Pareto-distributed risks with tail distortion parameter $\rho=1.2$.

Taking $\delta=1$ means that no distortion on the dependence structure is made, that is $C^{1}=C$, and $\pi_{\rho}^{1}[Z]=\pi_{\rho}[Z]$. In other words, the CDRM with $\delta=1$ reduces to Wang's DRM, which can be seen in the second columns of Tables 1 and 2. This fact is also clear in Figure 2. On the other hand, as $\delta$ increases, the transformed Kendall's tau decreases meaning that the dependence gets weaker (see the second lines of Tables 1 and 2). Moreover, starting from some $\delta$ the CDRM values become roughly constant while being always greater than the expectation (see the third lines of Tables 1 and 2).

\section{Concluding remarks}

In portfolio analysis, the dependence structure has a major role to play when quantifying risks. This led us to think of risk measure taking into account this fact in addition to the tail behavior. In this paper, we proposed a risk measure for the sum of two dependent losses by simultaneously transforming the distribution tail and the copula, which represents the dependence between the margins, by means of two distortion functions. We obtained a coherent measure that we called the Copula Distorted Risk Measure. This new measure has the characteristic to be greater than the expectation and less than the popular Wang's DRM. In the insurance business, the main advantage of this property is to reduce Wang's premium while respecting the standard axioms of the premium principle.

\section{Acknowledgment}

The authors would like to thank the Editors and the anonymous reviewers for their comments and suggestions that improved the quality of the paper.

\section{References}

[1] Artzner, P., Delbaen, F., Eber, J.M., Heath, D., 1999. Coherent measures of risk. Mathematical Finance 9, $203-228$.

[2] Burgert, C., Rüschendorf, L., 2006. Consistent risk measures for portfolio vectors. Insurance Math. Econom. 38, $289-297$.

[3] Crane, G., van der Hoek, J., 2008. Using distortions of copulas to price synthetic CDOs. Insurance Math. Econom. 42, 903-908.

[4] Darkiewicz, G., Dhaene, J., Goovaerts, M.J., 2004. Distortion risk measures for sums of random variables. Blätter der DGVFM, Springer Berlin/Heidelberg 26, 631-641.

[5] Denneberg, D., 1994. Non-additive measure and integral. Theory and Decision Library 27, Kluwer Academic Publilshers.

[6] Denuit, M., Dhaene, J., Goovaerts, M. and Kaas, R., 2005. Actuarial Theory for Dependent Risks : Measures, Orders and Models. Jhon Wiley \& Sons, Ltd.

[7] Durrleman, V., Nikeghbali, A., Roncalli, T., 2000. A simple transformation of copulas. Groupe de Recherche Opérationnelle, Crédit Lyonnais, France. 
[8] Frees, W.E., Valdez, E.A., 1998. Understanding relationships using copulas. N. Am. Actuar. J. 2, 1-25.

[9] Furman, E., Zitikis, R., 2008a. Weighted premium calculation principles. Insurance Math. Econom. 42, 459-465.

[10] Furman, E., Zitikis, R., 2008b. Weighted risk capital allocations. Insurance Math. Econom. 43, 263-269.

[11] Genest, C., Rivest, L.P., 2001. On the multivariate probability integral transformation. Statist. Probab. Lett. 53, 391-399.

[12] Hürlimann, W., 1998. On stop-loss order and the distortion pricing principle. ASTIN Bulletin 28, 119-134.

[13] Morillas, P.M., 2005. A method to obtain new copulas from a given one. Metrika 61, 169-184.

[14] Nelsen, R., 2006. An introduction to copulas. Springer Verlag, New York.

[15] Sklar, A., 1959. Fonctions de répartition à n dimensions et leurs marges. Inst. Statist. Univ. Paris 8, $229-231$.

[16] Valdez, E.A., Xiao, Y., 2010. On the distortion of a copula and its margins. Scand. Actuar. J. (in press).

[17] Wang, S.S., 1995. Insurance pricing and increased limits ratemaking by proportional hazards transforms. Insurance Math. Econom., 17, 43-54.

[18] Wang, S.S., 2000. A class of distortion operators for pricing financial and insurance risks. Journal of Risk and Insurance 67, $15-36$. 